\title{
Impact of a New Strategy of Deployment Diesel Generators on a Power System under Contingency
}

\author{
Firas M. Tuaimah \\ University of Baghdad \\ College of Engineering \\ Electrical Department
}

\begin{abstract}
This article presents an analysis of a power system under different types of contingences (power generators and high voltage transmission line outages). The work initiated by a new strategy based on location of deployment distributed generators, namely scenario A and scenario B. System losses, line loading capability and generated power has been determined, with and without the existence of the distributed generators deployment in the distribution network. A Diesel Generators with 5MVA each has been chosen to be as a distributed generators for many of economical considerations, the major is that they can be set to switch on when there's interference in power from the service organization and turn back off when power is reestablished for a safe. An arbitrary locations has been proposed as contingencies. The analysis done based on Newton Raphson and a linear programming approach as an Optimal Power Flow (OPF) applied on IEEE 30 bus test system. Matlab and Power World programs has been adopted for the simulation.
\end{abstract}

\section{Keywords}

Power flow, OPF, Diesel Generator, Contingency analysis

\section{INTRODUCTION}

The electric utilities are seeking for the advancements and technologies that are new to provide worthy control quality and unwavering quality to their customers.

Distributed Generators (DG) is considered to be as "taking energy to the load"". Distributed Generators guarantees to produce power with high efficiency and low contamination. DG evaluations run from $5 \mathrm{~kW}$ up to $100 \mathrm{MW}$. Maintenance cost for the DG, energy components and photovoltaic's is very low on account of the nonappearance of the moving parts.

A several late advancements have empowered the passage of power generation and vitality stockpiling at the distribution level. A portion of the real ones are recorded below .

a) Retail rivalry brought upon by utility structure.

b) With extended decision, customers and clients are requesting redone power supplies to suit their necessities

c) Need to concede substantial capital interests in building new focal station power plants and transmission lines

d) Appearance of a few innovations with diminished ecological effects and high change efficiencies

e) Appearance of productive and financially power electronic interfaces to enhance dependability and power quality

f) Capacity to adequately control various parts and subsystems utilizing state of the art PCs to oversee loads, requests, power flow, costumer's necessities
A few DG innovations are under different phases of advancement and developments. They incorporate photovoltaic systems (PV), micro turbines, wind energy conversion systems (WECS), gas turbines, gas fired IC engines, diesel engines, and fuel cell systems

Reconciliation of DG into a current utility can bring about a few benefits. These advantages incorporate reduction in line losses, diminished ecological effects, crest shaving, expanded general vitality proficiency, diminished transmission and circulation blockage, voltage booster, conceded speculations to update existing generation, transmission, and transmission system. [1,2]

[3] Presents a new approach for optimal operation of distribution networks with regard to Distributed Generators (DGs) based on metaheuristic techniques, These methods are tested on two examples distribution network containing an IEEE 34 distribution test feeders and a practical distribution system.

[4] Presents another procedure for deciding ideal portion and estimating of inserted generator in a conveyance system. The proposed method was tried on the 69 bus dissemination system and the outcomes demonstrated a noteworthy diminishment in circulation misfortunes and voltage profile enhancement.

[5] Designed a strategy based on a proposed "critical bus tracking" method for Proton Exchange Membrane Fuel Cell (PEMFC) DG is tested on a modified IEEE 14 bus test case. Matlab Distributed Computing System (MDCS) is applied for a reduced computation time. Program for contingency analysis is also implemented in MDCS to test the design strategy.

[6] Discussed an assessment of wind penetration effects on the power system transient stability. The wind generators considered are the squirrel cage induction generator (SCIG), which is a type of DG

[7] Provides an approach to calculate the new energy's maximum installation capability and the optimal installation site for regional power network Basing on the analysis of stochastic models of wind power, solar output and load.

[8] The focal point of this paper is on the design of a distributed energy management methodology system (EMS) for the ideal activity of microgrids with thought of the distribution organize and the related imperatives. In particular, a plan of microgrid energy administration as an ideal power flow issue, and propose a disseminated EMS where the microgrid central controller MGCC and the neighborhood controllers mutually figure an ideal schedule.

This paper centers around line loses under different contingencies. In this study a diesel generator which a distributed generator will be matched in the proposed 
network, namely IEEE bus test system, to investigate their effect.

\section{CONTINGENCY ANALYSIS IN DEREGULATED POWER SYSTEM \\ [1]}

As a result of un-planned power exchange to meet the power utilizations because of development of power system, the lines of the transmission system are required to work relatively equivalent or past as far as possible to the thermal limits.

So there might be an issue of over loading in some of the lines in the transmission system of some truly paths that are loaded, which may leads to emergency (contingency), if the transferred power of the transmission lines concerned isn't intelligently controlled.

Under emergency situation the power system can reach dangerous point. Generally, the contingencies can be in two types given below.

- $\quad$ The Outage of Transmission lines

- Generator Outage with bus, or without the bus

The contingency analysis defines as one of the analytical tools that are widely used to find the effect of the pre mentioned outages in the security study. The outage of line might increase the total power loss in any power system and it might lead to outage of more lines in the transmission system cascading which might collapse the system to a case or blackouts.

Violations caused because of the above possibilities are depicted underneath.

\subsection{Load Voltage Violations}

At each bus the voltage supposed to be 1 per unit. The acceptable voltage variety restrain is $\pm 10 \%$. i.e. $0.9-1.1$ p.u. Now if the voltage at any bus becomes below 0.9 p.u that is known as the low voltage. Additionally if the voltage value becomes higher than $1.1 \mathrm{p} . \mathrm{u}$ that is the high voltage profile.

\section{2 Line Thermal Limits Violations}

The actual MVA transferred becomes more than the MVA rating of the transmission line this leads to an emergency case. Fundamentally the transmission lines can hold out up to $125 \%$ of its designed MVA maximum thermal limit. If the transfered power limit exceeds $90 \%$ of the evaluated value, it might be dealt with as caution circumstance.

\section{DIESEL GENERATOR}

\section{DEPLOYMENT STRATEGY}

Two straightforward spiral systems are given as in below:

a) System without diesel generator (DG).

b) System with the consideration of diesel generator (DG) in the load buses.

The two proposed systems have a gathered load at the end of the line. The length of the line is thought to be $\mathrm{L}$ in $\mathrm{km}$. Schematics of proposed strategy adopted in this article for the above second case with two scenarios. Figure. 1 shows the system without DG.

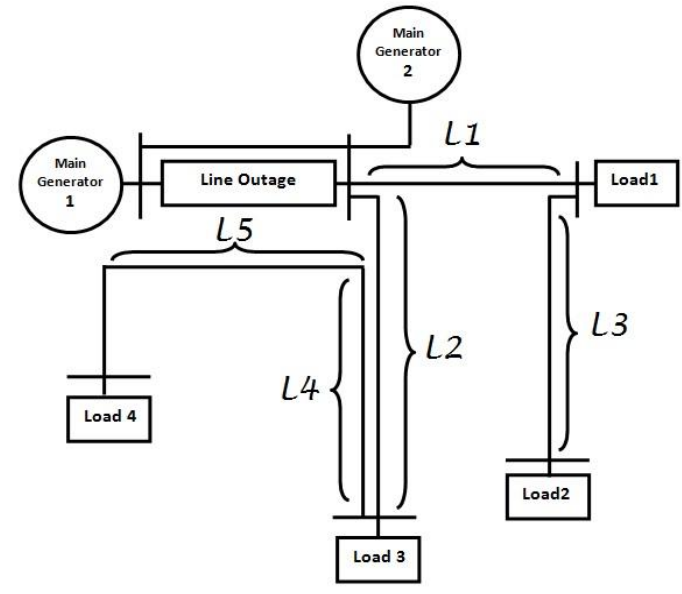

Fig 1: System without DG case

The first scenario (scenario A) the DG's will be deployed in the power system starting from load buses far from the location of the contingency case depending on the lengths as shown in Figure 2. In which DG1 is the furthest one from the contingency case (line outage) and DG4 is the nearest one.

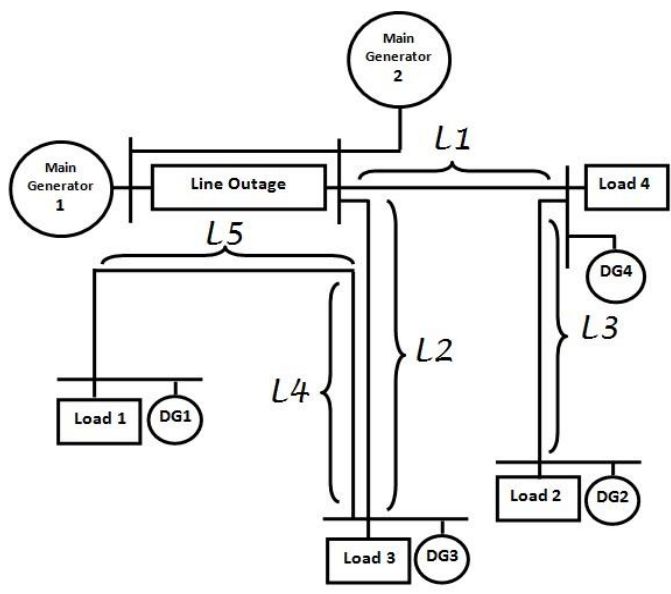

Fig 2: Scenario A for line outage case

For the second scenario (scenario B) the DG's will be deployed in the power system starting from load buses near from the location of the contingency case depending on the lengths as shown in Figure 3. In which DG1 is the nearest one from the contingency case (line outage) and DG4 is furthest one.

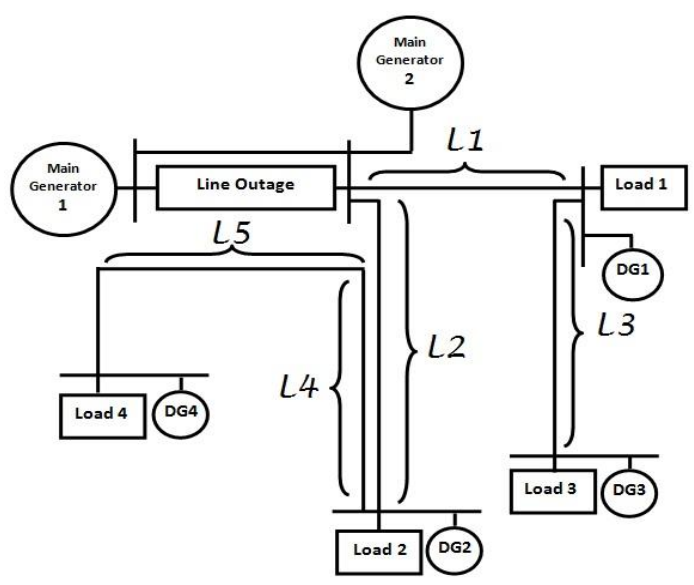

Fig 3: Scenario B for line outage case 
The same two scenarios will be adopted in this article for generator outages, Figure 4 shows the flowchart that be followed to evaluate the line losses for the above two scenarios.

Depending on above two scenarios the DG insertion strategy based on the transmission line lengths will be as in Table 1 below:

Table 1: DG sequence Vs. Lengths for Scenario A and B

\begin{tabular}{|c|c|c|c|c|c|c|c|}
\hline & $D G 1$ & $D G 2$ & $D G 3$ & $D G 4$ & $D G 5$ & $D G(1+1)$ & $D G n$ \\
\hline ScenarioA & $12+11+15$ & $11+13$ & 12 & 11 & $1(D G 5 \cdot 10)$ & $1(D G(i+1)-10)$ & $1(D G(n)-10)$ \\
\hline Scenario B & 11 & 12 & $11+13$ & $12+14+15$ & $1(D G 5 \cdot 10)$ & $1(D G(1+1)-10)$ & $1(D G(n)-10)$ \\
\hline
\end{tabular}

Where; $(12+14+15)>(11+13)>(12)>(11)>1($ DG5-lo $)>$ l(DG(i+1)- lo $)>1(\mathrm{DG}(\mathrm{n})-\mathrm{lo}), \mathrm{lo}=$ line outage

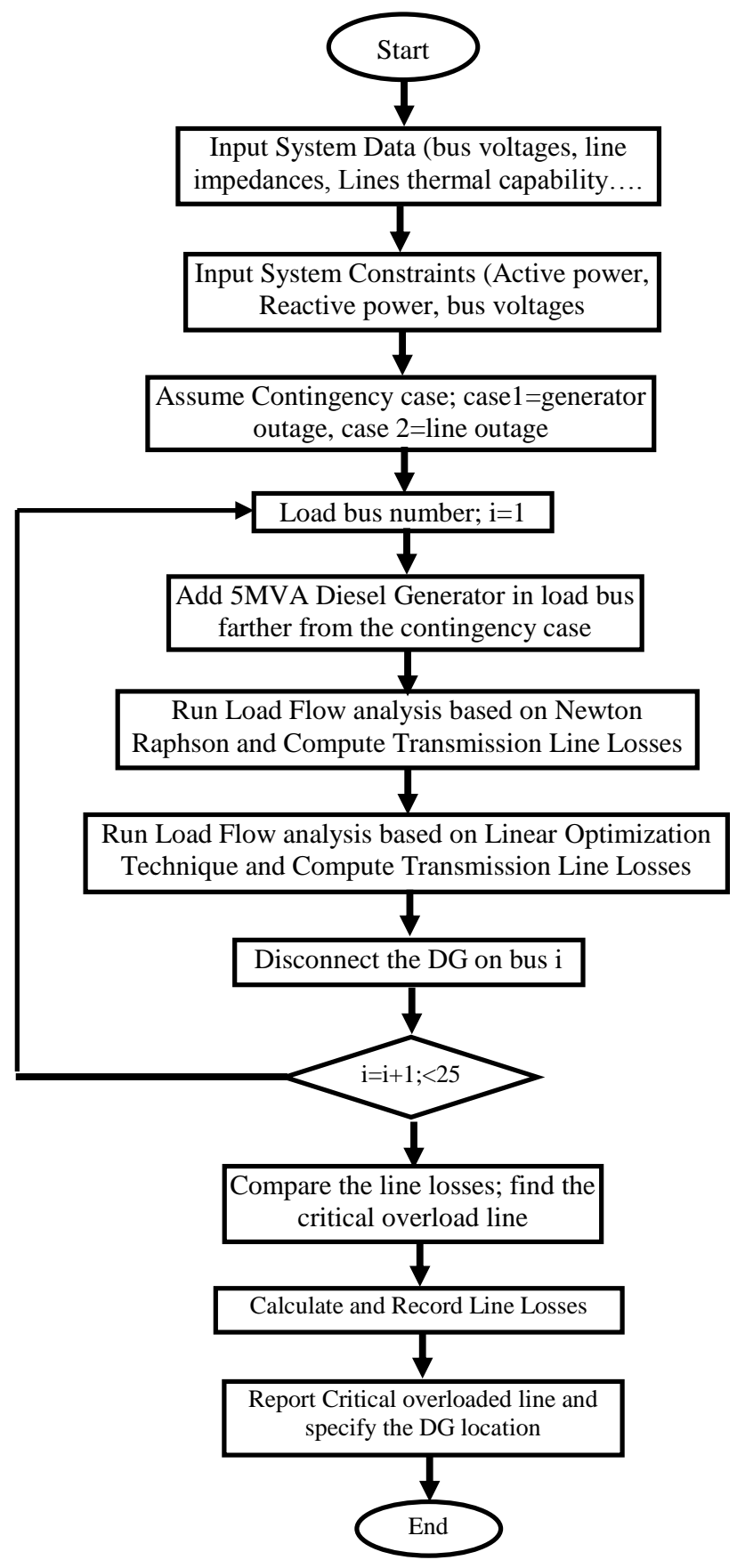

Fig 4: Proposed Methodology Flowchart

\section{DIESEL GENERATORS $[9,10]$}

Diesel generators include by far most of prime movers for standby power generators in view of their unwavering quality, solidness and execution under load. Diesel powered generators are relied upon for back up power systems in the most critical areas: hospitals centers, air terminals, government structures, media communications offices, and even atomic control plants. In standby power applications, diesel generators can begin and accept full-evaluated load in less than 10 seconds, and they ordinarily can go 30,000 hours or more between real overhauls.

A diesel generator is the mix of a diesel motor with an electric generator (regularly an alternator) to produce electrical vitality. This is a particular instance of motor generator. Table 2 shows the approximate price for a diesel engine with other types of DG types per kilowatt.

Table 2: Distributed Generators Approximate prices per kilowatt

\begin{tabular}{|l|l|l|}
\hline Technology & Size Range $(\mathbf{k W})$ & $\begin{array}{l}\text { Approximate } \\
\text { Installed Cost } \mathbf{( \$ / k W )}\end{array}$ \\
\hline Diesel engines & $20-10000$ & $125-300$ \\
\hline Turbine generator & $500-25000$ & $450-870$ \\
\hline Wind turbines & $10-1000$ & Around 1000 \\
\hline Microturbines & $30-200$ & $350-750$ \\
\hline Fuel cells & $50-2000$ & $1500-3000$ \\
\hline Photovoltaic & $<1-100$ & Around 3000 \\
& & \\
\hline
\end{tabular}

\section{OPTIMAL POWER FLOW CONSTRAINTS $[3,5,6]$}

The uniformity constraints $\mathbf{h}(\mathbf{x}, \mathbf{u})$ presents the static load flow conditions, for example, in the Kirchhoff's law $\forall \mathrm{b} \in \mathrm{B}$, where $\mathrm{B}$ is the arrangement of buses (indexed by $b$ ), and in the Kirchhoff's voltage law $\forall 1 \in \mathrm{L}$, where $\mathrm{L}$ is the arrangement of lines (indexed by 1 ).

The imbalance constraints $\mathbf{g}(\mathbf{x}, \mathbf{u})$ are recorded in the following .

(1) Active and reactive power limitations for the interconnection to the outside system (slack bus) $\forall_{\mathrm{O}} \in \mathrm{N}$

$$
\begin{aligned}
& P_{O}^{-} \leq P_{O} \leq P_{O}^{+} \\
& Q_{O}^{-} \leq Q_{O} \leq Q_{O}^{+}
\end{aligned}
$$

Where $\mathrm{N}$ is the arrangement of external sources (indexed. by o), $P_{O}$ are active output power, $Q_{O}$ are the reactive output power of n, respectively, and $P_{O}^{-} \leq P_{O}^{+}$and $Q_{O}^{-} \leq Q_{O}^{+}$are the $\min /$ max assumed values.

(2) Active and receptive power requirements for the diesel generators $\forall \mathrm{g} \in \mathrm{N}_{\mathrm{G}}$

$$
\begin{gathered}
P_{g}^{-} \leq P_{g} \leq P_{g}^{+} \\
Q_{g}^{-} \leq Q_{g} \leq Q_{g}^{+}
\end{gathered}
$$

Where $P_{g}$ and $Q_{g}$ are the active power and reactive power of the diesel generator $\mathrm{g}$, respectively, and receptive power yields of g, individually, and $P_{g}^{-} \leq P_{g}^{+}$and $Q_{g}^{-} \leq Q_{g}^{+}$are the $\min / \max$ assumed values.

3) Voltage level constraints $\forall b \in B$ 


$$
V_{b}^{-} \leq V_{b} \leq V_{b}^{+}
$$

Where $V_{b}$ is the voltage at $b, V_{b}^{+}$is the max. value that can be assumed and $V_{b}^{-}$is the min. value that it can be assumed.

4) Flow constraints for the lines and transformers $\forall l \in L$

$$
\sqrt{\left(f_{l}^{P}\right)^{2}+\left(f_{l}^{Q}\right)^{2}} \leq f_{l}^{+}
$$

Where $f_{l}^{P}$ represent the active power injected onto $l$ and $f_{l}^{Q}$ represent the reactive power injections onto $l$, and $f_{l}^{+}$is the maximum power flow on $l$.

\section{LINE LOSS DIMINISHMENT EXAMINATION}

The extent of the losses relies upon sum current stream and the line protection. In this way, line losses can be diminished by lessening either the current in the line, resistance or both. In the event that DG is utilized to give vitality locally to the load, line losses can be decreased in light of the abatement in current flow in some piece of the system .

Line losses equation for a three phase system can be derived as:

$S_{3 \emptyset}=\sqrt{3} V_{L} I_{L}^{*}$

Since

$S_{3 \emptyset}=P_{L}+j Q_{L}$

From eq. 1 and eq. 2 it can be concluded that:

$I_{L}=\frac{P_{L}-j Q_{L}}{\sqrt{3} V_{L}}$

Where $P_{L}$ and $Q_{L}$ are the load active power and reactive power, respectively

Since each transmission line is a combination of resistance and reactance and so can be given as

$$
Z_{\mathrm{L}}=R_{L}+j X_{L}
$$

Where $R_{L}$ and $X_{L}$ Line resistance and reactance per phase per unit length, respectively, $\Omega / \mathrm{km}$

The transmission line losses

$T_{\mathrm{L}-\mathrm{LOSSES}}=I_{L}^{2} Z_{L} l=I_{L}^{2}\left(R_{L}+j X_{L}\right) l$

Where $l$ is the line length in $\mathrm{km}$, Then the transmission line losses will be given as:

$T_{\mathrm{L}-\mathrm{L} O S S E S}=\frac{P_{L}^{2}+Q_{L}^{2}}{3 V_{L}^{2}} Z_{L} l=\left(\frac{P_{L}^{2}+Q_{L}^{2}}{3 V_{L}^{2}}\right)\left(R_{L}+j X_{L}\right) l$

For simplification $\mathrm{X}_{\mathrm{L} \approx 0}$, then

$T_{\mathrm{L}-\mathrm{LOSSES}}=\left(\frac{P_{L}^{2}+Q_{L}^{2}}{3 V_{L}^{2}}\right) R_{L} l$

\subsection{Transmission Line losses Computation for System without DG}

Referring to Figure 1, the line losses between load 1 and the bus that is near to the line outage contingency is 11 in $\mathrm{km}$, Assuming a short transmission line, neglecting the voltage drop along the line and accordingly the losses can be calculated as:

$T_{\mathrm{L}-\mathrm{L} O S S E S-O 1}=\left(\frac{P_{L o 1}^{2}+Q_{L o 1}^{2}}{3 V_{L}^{2}}\right) R_{L o 1} \times 11$

Then the full system losses can be calculated as:

$$
\begin{aligned}
& T_{\mathrm{L}-\mathrm{LOSSES}}=\left(\frac{P_{L o 1}^{2}+Q_{L O 1}^{2}}{3 V_{L}^{2}}\right) R_{L o 1} \times 11+\left(\frac{P_{L o 2}^{2}+Q_{L o 2}^{2}}{3 V_{L}^{2}}\right) R_{L o 2} \times \\
& (11+13)+\left(\frac{P_{L O 3}^{2}+Q_{L o 3}^{2}}{3 V_{L}^{2}}\right) R_{L o 3} \times(12)+\left(\frac{P_{L O 4}^{2}+Q_{L o 4}^{2}}{3 V_{L}^{2}}\right) R_{L o 4} \times \\
& (12+14+15)
\end{aligned}
$$

And so on for larger power network

\subsection{Transmission Line Losses Computation for System with DG}

The DG output current can be calculated as:

$$
I_{G}=\frac{P_{G}-j Q_{G}}{\sqrt{3} V_{L}}
$$

Accordingly the current flow in the line will be reduced to:

$I_{L O A D}=I_{L}-I_{G}=\frac{P_{L}-j Q_{L}}{\sqrt{3} V_{L}}-\frac{P_{G}-j Q_{G}}{\sqrt{3} V_{L}}=\frac{\left(P_{L}-P_{G}\right)-j\left(Q_{L}-Q_{G}\right)}{\sqrt{3} V_{L}}$

The line losses can be given as:

$$
T_{\mathrm{L}-\mathrm{L} O S S E S}=\frac{P_{L}^{2}-2 P_{L} P_{G}+P_{L}^{2}+Q_{L}^{2}-2 Q_{L} Q_{G}+Q_{G}^{2}}{3 V_{L}^{2}} R_{L} l
$$

\section{a. (Scenario A), Addition of a DG starting from furthest load bus:}

Referring to Figure 2, the line losses between load 1and the bus that is near to the line outage contingency is $12+14+15$ in $\mathrm{km}$, assuming that the transmission line is short, neglecting the voltage drop along the line and the losses can be calculated as:

$T_{\mathrm{L}-\mathrm{LOSSES}-04}=\frac{P_{\mathrm{L} 04}^{2}-2 P_{L 04} P_{G}+P_{\mathrm{LO4}}^{2}+Q_{\mathrm{LO} 4}^{2}-2 Q_{\mathrm{LO} 4} Q_{G}+Q_{G}^{2}}{3 V_{L}^{2}} R_{L O 4} \times$
$(12+14+15)$

\section{b. (Scenario B), Addition of a DG starting from nearest load bus:}

Referring to figure 3 , the line losses between load 1 and the bus that is near to the line outage contingency is 11 in $\mathrm{km}$, assuming that the transmission line is short, neglecting the voltage drop along the transmission line, and the losses can be calculated as:

$T_{\mathrm{L}-\mathrm{LOSSES}-o 1}=\frac{P_{L o 1}^{2}-2 P_{L o 1} P_{G}+P_{L o 1}^{2}+Q_{L o 1}^{2}-2 Q_{L o 1} Q_{G}+Q_{G}^{2}}{3 V_{L}^{2}} R_{L o 1} \times 11$

\section{CASE STUDY}

In order to compute the transmission line losses, active and reactive power flow, the two pre mentioned scenarios methodologies will be tested on IEEE 30 bus system shown in Figure 5, the basic system data are given in Table 2 and Table 3.

The MATLAB programming language and Power World program will be proposed as simulation programs for comparison.

The load flow analysis for the IEEE 30 bus test will be modeled based on optimal power flow depending on two of the conventional methods, namely Newton-Raphson and linear programming. 
Table 2: IEEE 30 Bus Test System Data [11]

\begin{tabular}{|l|l|}
\hline Generators & 6 \\
\hline Buses & 30 \\
\hline Load points & 21 \\
\hline Lines & 41 \\
\hline \multicolumn{1}{|c|}{ Total generation capacity } & $435 \mathrm{MW}$ \\
\hline Fixed load & $283.4 \mathrm{MW}$ \\
\hline
\end{tabular}

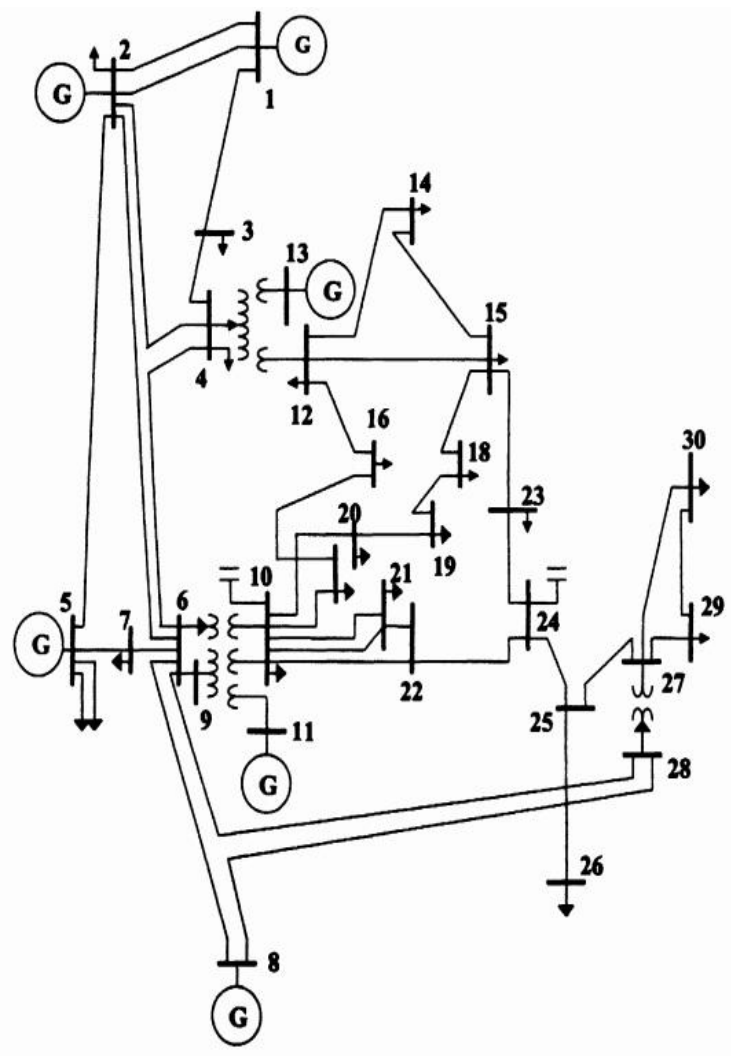

Fig 5: IEEE 30 Bus Test System

Table 3: IEEE 30 Bus Test System maximum Line MVA Capability Thermal Rating [11]

\begin{tabular}{|l|l|l|l|}
\hline Line & Max. MVA & Line & Max. MVA \\
\hline $1-2$ & 130 & $15-18$ & 16 \\
\hline $1-3$ & 130 & $18-19$ & 16 \\
\hline $2-4$ & 65 & $19-20$ & 32 \\
\hline $3-4$ & 130 & $10-20$ & 32 \\
\hline $2-5$ & 130 & $10-17$ & 32 \\
\hline $2-6$ & 65 & $10-21$ & 32 \\
\hline $4-6$ & 90 & $10-22$ & 32 \\
\hline $5-7$ & 70 & $21-22$ & 32 \\
\hline $6-7$ & 130 & $15-23$ & 16 \\
\hline $6-8$ & 32 & $22-24$ & 16 \\
\hline $6-9$ & 65 & $23-24$ & 16 \\
\hline
\end{tabular}

\begin{tabular}{|l|l|l|l|}
\hline $6-10$ & 32 & $24-25$ & 16 \\
\hline $9-11$ & 65 & $25-26$ & 16 \\
\hline $9-10$ & 65 & $25-27$ & 16 \\
\hline $4-12$ & 65 & $28-27$ & 65 \\
\hline $12-13$ & 65 & $27-29$ & 16 \\
\hline $12-14$ & 32 & $27-30$ & 16 \\
\hline $12-15$ & 32 & $29-30$ & 16 \\
\hline $12-16$ & 32 & $8-28$ & 32 \\
\hline $14-15$ & 16 & $6-28$ & 32 \\
\hline $16-17$ & 16 & & \\
\hline
\end{tabular}

\section{SIMULATION AND RESULTS}

To investigate the effectiveness of the proposed strategy based on two scenarios, three case studies has been suggested as a contingencies, namely; generator 2 outage, line 2-4 outage and line 2-6 outage and for all the cases, the MW load has been sustained to 283.4 and MVar load has been also sustained to 126.2. The results for all cases will be given below:

Case 1: is the healthy case where there is no any contingency. Accordingly the generated MW and MVar are listed in Table 4; also the transmission line MW and MVar losses are given. In which the reduction in active power losses between adopting OPF using Matlab programing over the Newton Raphson is $40.7 \%$, while when using the Power World simulation program is $37.7 \%$. The above extracted results are close to each other when adopting the two programming languages.

Case 2: is the case where generator 2 are being outage, accordingly the generated MW and MVar are listed in Table 5, also the transmission line MW and MVar losses are given, in which bus 26 is calculated to be furthest bus to generator 2 whereas bus 4 is the nearest to generator 2. i.e. 5 MVA diesel generator will be added at buses 26 and 4 . In which the reduction in active power losses between adopting OPF using Matlab programing over the case without using DG for scenario $\mathrm{A}$ is $2.7 \%$ and for scenario $\mathrm{B}$ is $1 \%$. The above extracted results show that scenario $\mathrm{A}$ is better than scenario $\mathrm{B}$ in losses reduction.

Case 3: is the case where line 2-6 are being outage, accordingly the generated MW and MVar are listed in Table 6 , also the transmission line MW and MVar losses are given, in which bus 29 is calculated to be furthest bus to line $2-6$ whereas bus 6 is the nearest to line 2-6. i.e. 5 MVA diesel generator will be added at buses 29 and 6 . In which the reduction in active power losses between adopting OPF using Matlab programing over the case without using DG for scenario $\mathrm{A}$ is $2.73 \%$ and for scenario $\mathrm{B}$ is $1.6 \%$. The above extracted results show that scenario $\mathrm{A}$ is better than scenario $\mathrm{B}$ in losses reduction.

Case 4: is the case where line 2-4 are being outage, accordingly the generated MW and MVar are listed in Table 7 , also the transmission line MW and MVar losses are given, in which bus 28 is calculated to be furthest bus to line 2-4 whereas bus 4 is the nearest to line 2-4. i.e. 5 MVA diesel generator will be added at buses 28 and 4 . In which the 
reduction in active power losses between adopting OPF using Matlab programing over the case without using DG for scenario $\mathrm{A}$ is $1.37 \%$ and for scenario $\mathrm{B}$ is $1 . \%$. The above extracted results show that scenario A is better than scenario $\mathrm{B}$ in losses reduction.

Table 4: MW and MVar for Generated and Losses Based on Newton Raphson and OPF for Case 1

\begin{tabular}{|l|l|l|l|}
\cline { 2 - 4 } \multicolumn{1}{c|}{} & Newton Raphson & OPF based on Matlab & OPF based on Power World \\
\hline MW Generated & 300.998 & 293.83 & 294.35 \\
\hline MVar Generated & 125.144 & 107.14 & 111.79 \\
\hline MW Losses & 17.599 & 10.432 & 10.96 \\
\hline MVar Losses & 22.244 & 40.88 & 10.66 \\
\hline
\end{tabular}

Table 5: MW and MVar for Generated and Losses Based on Newton Raphson and OPF for Case 2

\begin{tabular}{|c|c|c|c|c|c|c|c|}
\hline & \multirow[b]{2}{*}{$\begin{array}{l}\text { Newton } \\
\text { Raphson }\end{array}$} & \multicolumn{3}{|c|}{ OPF based on Matlab } & \multicolumn{3}{|c|}{ OPF based on Power World } \\
\hline & & $\begin{array}{l}\text { Without } \\
\text { DG }\end{array}$ & Scenario A & Scenario B & $\begin{array}{l}\text { Without } \\
\text { DG }\end{array}$ & Scenario A & Scenario B \\
\hline MW Generated & 304.198 & 293.34 & 293.07 & 293.24 & 292.98 & 293.07 & 293.44 \\
\hline MVar Generated & 137.234 & 106.11 & 103.99 & 105.16 & 107.71 & 108 & 111.45 \\
\hline MW Losses & 20.8 & 9.936 & 9.672 & 9.84 & 9.58 & 9.67 & 10.04 \\
\hline MVar Losses & 34.351 & 39.3 & 37.93 & 38.92 & 6 & 5.99 & 8.62 \\
\hline
\end{tabular}

Table 6: MW and MVar for Generated and Losses Based on Newton Raphson and OPF for Case 3

\begin{tabular}{|c|c|c|c|c|c|c|c|}
\hline & \multirow[b]{2}{*}{$\begin{array}{l}\text { Newton } \\
\text { Raphson }\end{array}$} & \multicolumn{3}{|c|}{ OPF based on Matlab } & \multicolumn{3}{|c|}{ OPF based on Power World } \\
\hline & & $\begin{array}{l}\text { Without } \\
\text { DG }\end{array}$ & Scenario A & Scenario B & $\begin{array}{l}\text { Without } \\
\text { DG }\end{array}$ & Scenario A & Scenario B \\
\hline MW Generated & 303.742 & 294.87 & 294.55 & 294.69 & 296.01 & 295.51 & 295.73 \\
\hline MVar Generated & 142.345 & 116.2 & 114.42 & 115.4 & 124.98 & 123.02 & 125.33 \\
\hline MW Losses & 20.348 & 11.467 & 11.153 & 11.286 & 12.63 & 12.12 & 12.33 \\
\hline MVar Losses & 39.452 & 45.53 & 43.95 & 44.85 & 22.86 & 20.76 & 22.44 \\
\hline
\end{tabular}

Table 7: MW and MVar for Generated and Losses Based on Newton Raphson and OPF for Case 4

\begin{tabular}{|c|c|c|c|c|c|c|c|}
\hline & \multirow[b]{2}{*}{$\begin{array}{l}\text { Newton } \\
\text { Raphson }\end{array}$} & \multicolumn{3}{|c|}{ OPF based on Matlab } & \multicolumn{3}{|c|}{ OPF based on Power World } \\
\hline & & $\begin{array}{l}\text { Without } \\
\text { DG }\end{array}$ & Scenario A & Scenario B & $\begin{array}{l}\text { Without } \\
\text { DG }\end{array}$ & Scenario A & Scenario B \\
\hline MW Generated & 302.45 & 294.37 & 294.22 & 294.26 & 295.23 & 294.82 & 294.89 \\
\hline MVar Generated & 136.595 & 113.67 & 113 & 113.22 & 121.03 & 119.73 & 119.99 \\
\hline MW Losses & 19.055 & 10.966 & 10.815 & 10.863 & 11.85 & 11.42 & 11.49 \\
\hline MVar Losses & 33.701 & 43.24 & 42.69 & 42.86 & 43.24 & 17.79 & 18.02 \\
\hline
\end{tabular}

Figure 6 shows the IEEE 30 Bus Test System configuration built in Power World simulation program, in which the following results are shown in the upper -right corner, the generated active and reactive power, the load active and reactive power and finally active and reactive power losses, respectively. The countor diagrams will be drawn for healthy case, generator 2 outage, line 2-6 outage and line 2-4 outage without DG and with DG's deployment in the nearest and farthest buses as shown in figures from 7 to 16 . The red zone shows the transmission line that carry power over the thermal capability of the lines. 


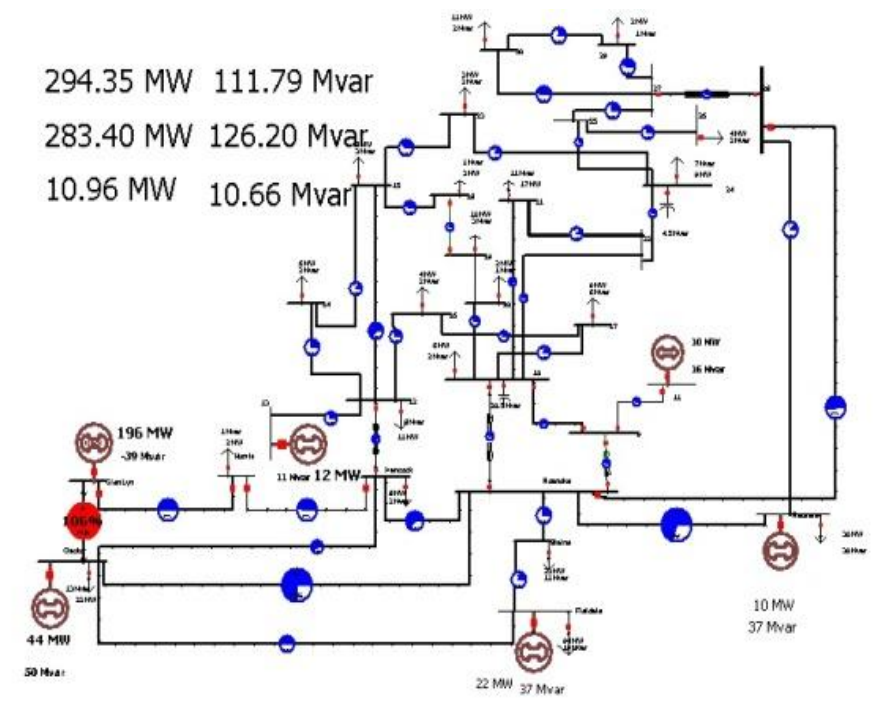

Fig 6: IEEE 30 Bus Test System

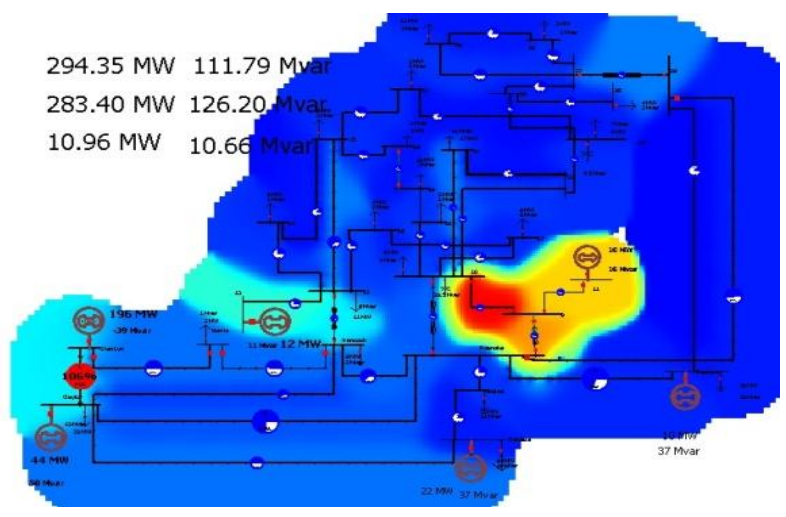

Fig 7: IEEE 30 Bus System contour for healthy case

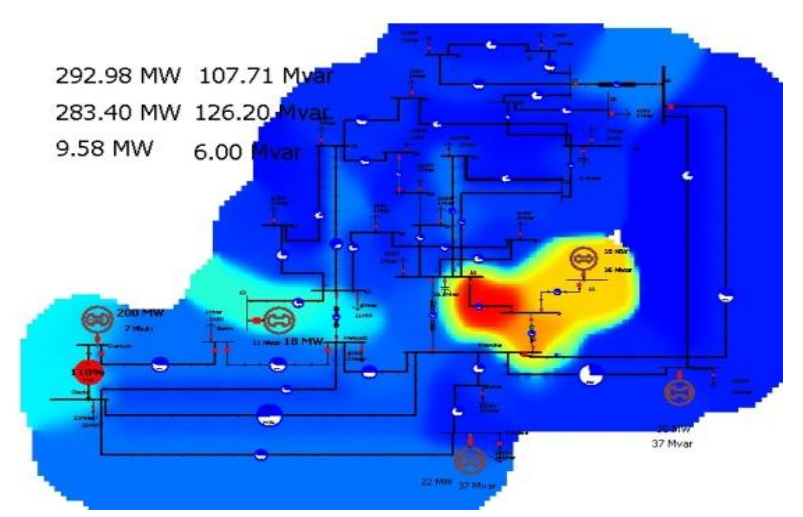

Fig 8: IEEE 30 Bus System contour when Generator 2 outage

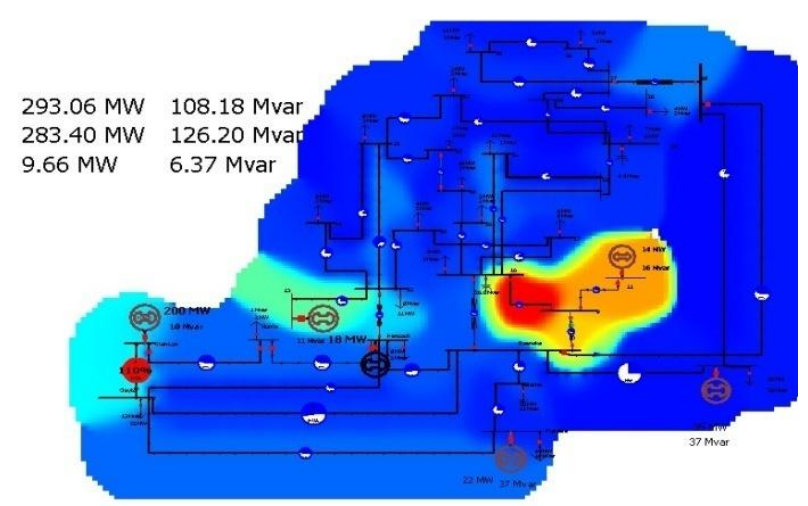

Fig 9: IEEE 30 Bus System contour when Generator 2 outage with 5MVA DG addition at bus 4

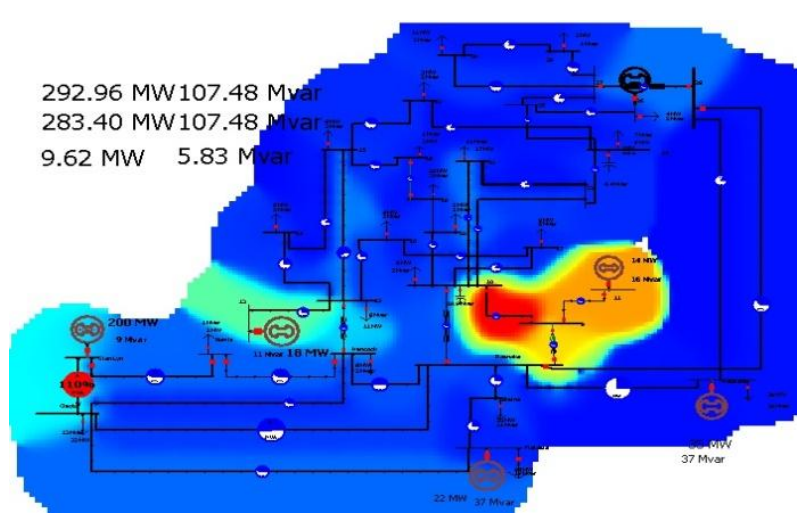

Fig 10: IEEE 30 Bus System contour when Generator 2 outage with 5MVA DG addition at bus 28 


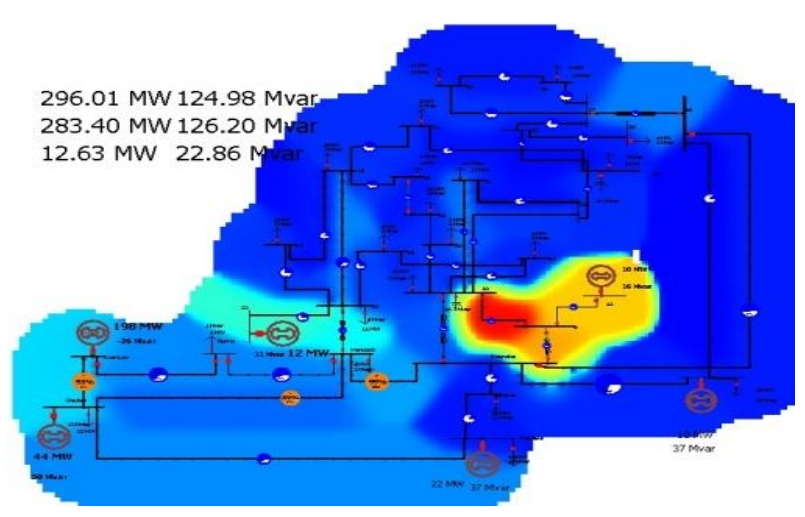

Fig 11: IEEE 30 Bus System contour when line 2-6 outage

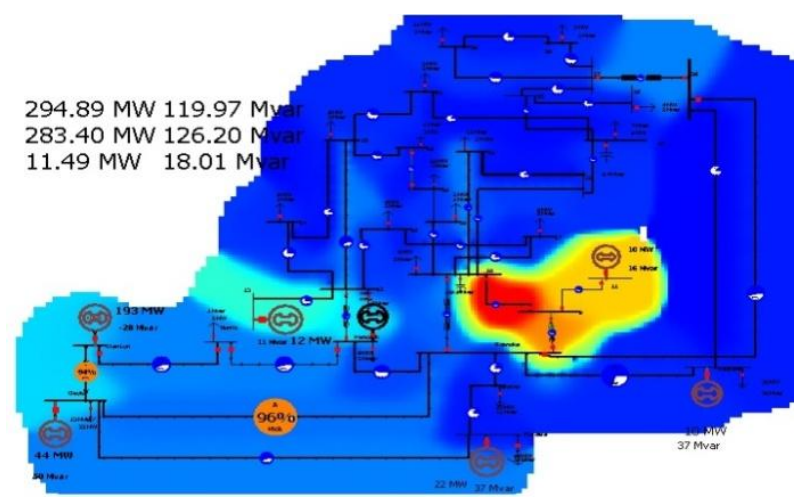

Fig 12: IEEE 30 Bus System contour when line 2-6 outage with 5MVA DG addition at bus 6

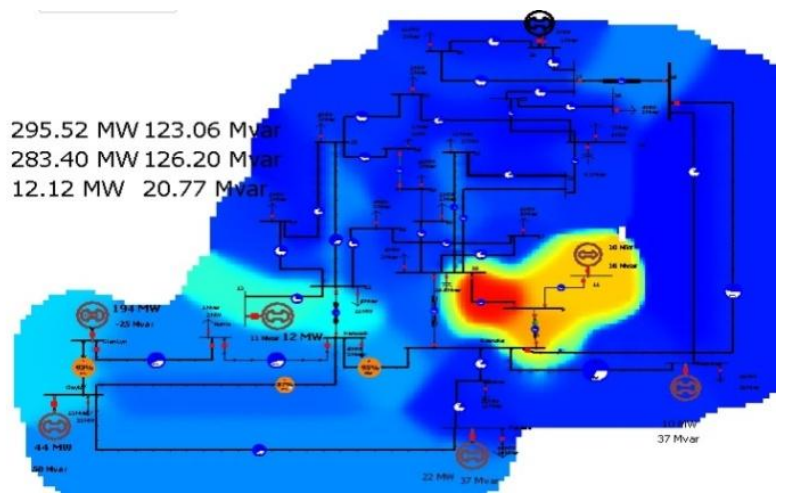

Fig 13: IEEE 30 Bus System contour when line 2-6 outage with 5MVA DG addition at bus 29

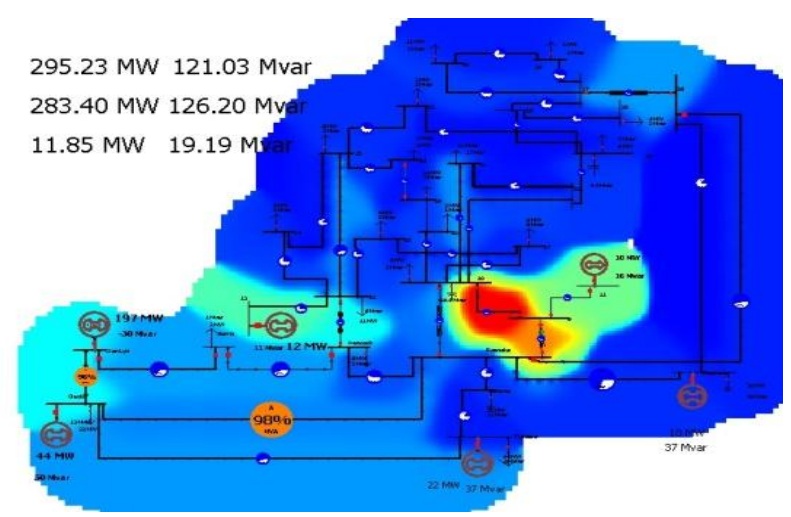

Fig 14: IEEE 30 Bus System contour when line 2-4 outage

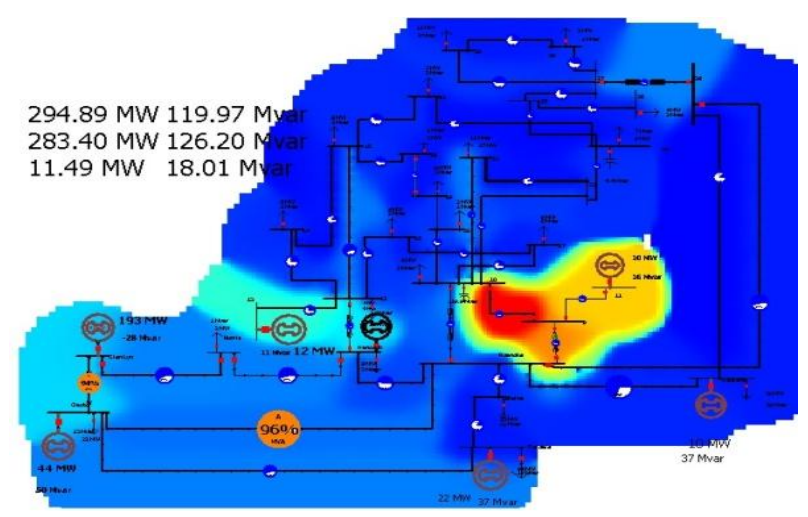

Fig 15: IEEE 30 Bus System contour when line 2-4 outage with 5MVA DG addition at bus 4

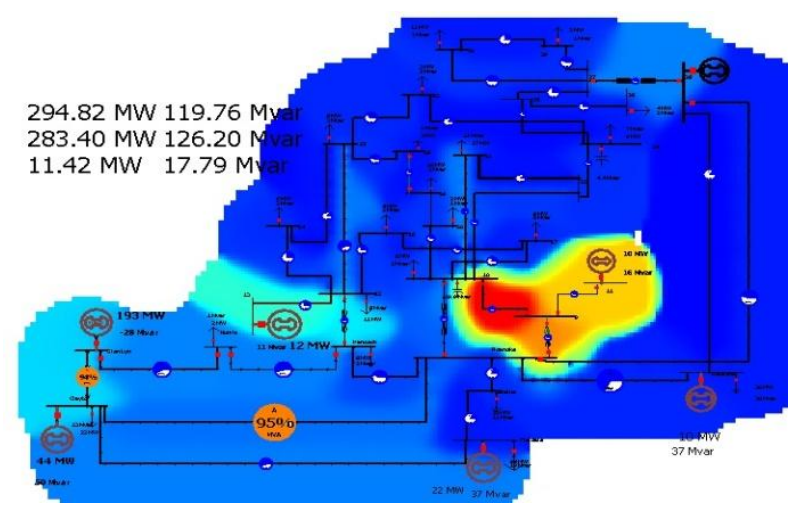

Fig 16: IEEE 30 Bus System contour when line 2-4 outage with 5MVA DG addition at bus 28

\section{CONCLUSION}

In this paper, Optimal Power Flow methodology has been adopted based on Linear Optimization Technique with a new strategy based on two scenarios to deploy the diesel generators at the instance of contingency. The deployment or distribution process balances the load requirements and tries to reduce the resulting active and reactive power losses. The algorithm has been applied on IEEE 30 bus test system. The results show that the reduction was larger in power losses when adopting scenario A rather than scenario B, which means that it is better to deploy the diesel generators starting from the far point from the contingency case. The results also show that there is close results obtained from applying the Matlab and Power World simulator.

\section{REFERENCES}

[1] Sushree Sangita Nayak, Swasti Bachan Panda and Sipra Das Mohapatra"State Estimation and Total Transfer Capability Calculation in Deregulated Power System" proceedings of the IEEE International Conference on Computing Methodologies and Communication (ICCMC), pp. 757-761, 2017

[2] Carlo Cecati, Costantino Citro, Antonio Piccolo and Pierluigi Siano"Smart Operation of Wind Turbines and Diesel Generators According to Economic Criteria" IEEE Transactions on Industrial Electronics, Vol. 58, No. 10, pp. 4514-4525, October 2011

[3] T. Niknam, A.M. Ranjbar, A.R. Shirani, B.Mozafari and A. Ostadi"Optimal Operation of Distribution System with Regard to Distributed Generation: A Comparison of Evolutionary Methods", IAS pp. 2690-2697, 2005 
[4] T.K.A. Rahman, S.R.A. Rahim and I. Musirin"Optimal Allocation and Sizing of Embedded Generators" National Power and Energy Conference (PECon) proceedings, Kuala Lumpur, Malaysia, pp. 288-294, 2004

[5] Wenzhong Gao and Xi Chen"Distributed Generation Placement Design and Contingency Analysis with Parallel Computing Technology", Journal of Computers, Vol. 4, No. 4, pp. 347-354, April 2009.

[6] Amroune Mohammed and Bouktir Tarek "Power System Transient Stability Analysis with High Wind Power Penetration"International Electrical Engineering Journal (IEEJ) Vol. 4, No.1,pp. 907-913, 2013

[7] Zhaoxia Sun, Weiwei Li, Jinfeng Zhu, Qiangmin Liu and Tianci Liu"A planning Method for sitting and Sizing of Distributed Generation Based on Chance-constrained Programming" 5th international conference on Electric
Utility Deregulation and Restructuring and Power Technologies, November 26-29, pp. 527-531, Changsha, China, 2015.

[8] Wenbo Shi, Xiaorong Xie, Chi-Cheng Chu, and Rajit Gadh"Distributed Optimal Energy Management in Microgrids" IEEE Transactions on Smart Grid, pp. 1- 10, 2014

[9] Natthaphob Nimpitiwan and Gerald Heydt"Consequences of Fault Currents Contributed by Distributed Generation"Power Systems Engineering Research Center, Arizona State University, 2006

[10] Timothy A. Loehlein"Maintenance is one key to diesel generator set reliability"Technical information from Cummins Power Generation, 2007

[11] Hadi Saadat "Power System Analysis", McGraw-Hill Companies, 1999 